\title{
Gestión ambiental de tránsito: cómo la ingeniería de transporte puede contribuir a la mejoría del ambiente urbano
}

\begin{abstract}
This paper intwduces the concept of Environmental Traffic Management. The relevance of this paper lies in the fact that this concept is almost unknown in Latin America. However, the Environmental Traffic Management has the potential of improving urban environment. Firstly, the concepts of transport system, traffic, traffic management, congestion and degree of saturation are introduced. Then, the way in which the urban as well as environmental impacts can be predicted by means of the degree of saturation is shown. Considering Santiago de Chile as case studys alternative schemes for reducing the degree of saturation are explored. Finally, the main conclusion is stated: it is not necessay to expand road capacity for reducing traffic impacts. It is necessary, however, to change the scope of traffic problems: from reducing road congestion to increase urban quality.

Keywords: urban environment, transport, traffic.
\end{abstract}

\section{Resumen}

Este artículo presenta la noción de gestión ambiental de tránsito. Su impor tancia radica en que esta noción es poco conocida en el medio chileno y latinoamericano, pero posee el potencial de mejorar el ambiente urbano. Primero, se precisan los conceptos de sistema de transporte, tráfico, gestión de tránsito, congestión y grado de saturación. Luego, se demuestra cómo impactos urbano-ambientales derivados del tráfico pueden ser cuantificados y predichos en función del grado de saturación. Tomando como ejemplo la ciudad de Santiago de Chile, se ilustran alternativas para reducir el grado de saturación. Se concluye que, de aplicarse ciertas medidas, los impactos derivados del tráfico podrían disminuir, sin necesidad de aumentar la capacidad vial. Es necesario, no obstante, cambiar la visión de los problemas de tráfico: desde r educir la congestión a incrementar la calidad urbana.

Palabras claves: medio ambiente urbano, transporte, tránsito. 


\section{Introducción}

$\mathrm{E}$ objetivo primario de este artículo es mostrar cómo aspectos a veces olvidados por la ingeniería civil pueden ayudar a un mejor ambiente urbano, y no al revés. Este objetivo se pretende alcanzar definiendo un concepto nuevo para la ingeniería chilena y latinoamericana, pero bastante utilizado en países desarrollados: la gestión ambiental de tránsito. Esta es una estrategia de la ingeniería de transporte mediante la cual se intenta introducir aspectos de urbanismo y medio ambiente dentro de las medidas de ingeniería que se toman para solucionar problemas de transporte. Si bien su concepción data de 1960, su incorporación a la práctica ha tenido lugar durante la última década del siglo pasado en Europa.

Un objetivo secundario de este escrito consiste en mostrar al urbanista cómo se puede hacer uso de herramientas propias de la ingeniería civil (v.g. modelos matemáticos predictivos), para cuantificar impactos urbanos derivados del tráfico. Esta motivación nace del hecho, no poco común, de recibir desde el urbanismo propuestas para mejorar el transporte urbano que se contraponen a resultados teóricos y empíricos provenientes de investigaciones de más de medio siglo en temas de ingeniería de transporte. Un ejemplo es la constr ucción de autopistas urbanas para solucionar "la congestión"; concepto que se intentará precisar en este artículo.

El contenido y alcance del artículo se enumera a continuación. El Capítulo 2 resume las definiciones (y objetivos) del sistema de transporte, tránsito, gestión (ambiental) de tránsito, grado de saturación y congestión. Se formula también la dificultad a la cual se ve enfrentada la práctica de la ingeniería chilena para aplicar la estrategia de gestión ambiental de tránsito, así como algunas propuestas para sortear estas dificultades. Una forma de cuantificar objetivamente los impactos urbanos y ambientales derivados del tráfico es

\footnotetext{
* Ingeniero Civil (U. de Chile); DIC, MSc y PhD in Transport Studies (U. of London). Profesor Asistente, Departamento de Ingeniería Civil, Facultad de Ciencias Físicas y Matemáticas, Universidad de Chile. E-mail: rodferna@ing.uchile.cl

"I Ingeniero Civil y Diplomado en Seguridad de Tránsito (U. de Chile). Profesor Adjunto, Departamento de Ingeniería Civil, Facultad de Ciencias Físicas y Matemáticas, Universidad de Chile. E-mail: evalenza@ing.uchile.cl
}

presentada en el Capítulo 3. Se elige una forma de medición física, antes que económica, ya que los impactos son percibidos en tal carácter por las personas. El Capítulo 4 muestra, basado en datos recientes de la Encuesta Origen Destino de Viajes del Gran Santiago, un ejemplo de cómo la gestión de tránsito puede contribuir a reducir los impactos urbano-ambientales del tráfico, en contraposición con la práctica de aumento de la capacidad vial. Finalmente, el Capítulo 5 se encarga de entregar unas breves conclusiones sobre todo lo expuesto.

\section{Definiciones y problemas}

\subsection{Definiciones}

Desde Manheim (1984) es sabido que el objetivo de la ingeniería de transporte es entender la dinámica y actuar sobre el sistema de transporte, resumido en la Figura 1.

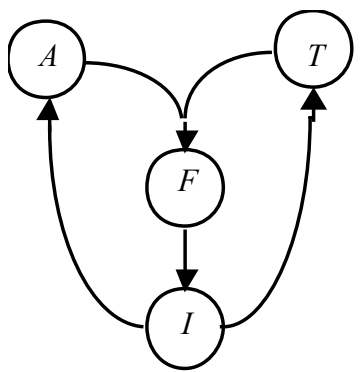

Figura 1. Esquema de Manheim.

Donde $A$ es el conjunto de actividades de residencia, producción, consumo, recreación, etc. en un área determinada (país, región, ciudad, comuna); $T$ es el conjunto de vías (red), vehículos, terminales (v.g. paraderos, estaciones) y su utilización en esa área; $F$ es el conjunto de viajes sobre la red entre diversos orígenes y destinos, en diferentes vehículos, por variadas rutas y en distintos períodos; e $I$ es el conjunto de efectos o impactos urbanos y ambientales derivados del tráfico sobre la red: congestión, polución, ruido, segregación, riesgo, intimidación, intrusión visual, inaccesibilidad al transporte público, etc. Dependiendo de la magnitud de estos impactos, las personas, en forma individual o colectiva (v.g. el Estado y sus instituciones), toman decisiones. En el mediano pla- 
zo, estas se manifiestan sobre el sistema de transporte (T); por ejemplo, aumento de frecuencias del transporte público, nueva línea de metro, aumento de la capacidad vial, etc. En un plazo mayor, las decisiones se observarán sobre el sistema de actividades $(A)$; por ejemplo, cambio en el lugar de residencia o trabajo, reubicación de actividades productivas, etc.

¿Cuál es, entonces, el problema que debe solucionar la ingeniería de transporte en el ambiente urbano? La respuesta es movilizar personas -y no vehículos- con eficiencia en cuanto a uso de los recursos, principalmente combustible y tiempo de las personas; uso del ambiente (aire, suelo urbano); seguridad -minimizando los accidentes en el tránsito-; y equidad social, con igual calidad para todos (al igual que los otros servicios públicos de distribución como agua, luz, gas, teléfono).

La expresión tangible de los viajes sobre la red $(F)$ es el tránsito o tráfico, entendido como la circulación de personas por los espacios públicos. En consecuencia, la gestión de tránsito es la administración racional del tráfico en un área urbana determinada. En su concepción moderna (ver por ejemplo IHT, 1997), el objetivo de la gestión de tránsito es aminorar la mayor cantidad de impactos urbanos y ambientales negativos derivados del tráfico. Es lo que hoy suele denominarse gestión ambiental de tránsito, para difer enciarla de aquella sólo centrada en vehículos, vías y congestión, nacida en la segunda mitad del siglo pasado. Luego, las palabras claves de las anteriores definiciones son:

- Racional. Requier e de una aproximación rigurosa; esta existe hace algún tiempo y es la Ingeniería de Tránsito, como especialización de la Ingeniería de Transporte.

- Personas. El centro del análisis debe ser las personas y no los vehículos; el hecho que parte de la circulación se realice en vehículos es una consecuencia de una urbe disgregada, no un fin en sí mismo.

- Espacio público. El medio ambiente del tráfico es el espacio público, no sólo las calzadas por donde circulan vehículos; incluye veredas, cruces y áreas peatonales, parader os, etc.

- Áreas. La gestión de tránsito se debe aplicar sobre zonas geográficas completas (barrio, comuna, ciudad) y no deben ser medidas aisladas espacial y temporalmente; es decir, la acción debe ser un conjunto de diseños en vez de medidas puntuales.
- Impactos urbanos. Debe considerar efectos que vayan más allá de la congestión vehicular, atacando todas las posibles consecuencias del tráfico sobre las personas.

Los impactos urbanos y ambientales asociados al tráfico son, en gran medida, dir ectamente proporcionales a una variable: el grado de saturación $(x)$ de cualquier dispositivo vial (vía, intersección, parader o, red). El grado de saturación se define como la razón entre el flujo que usa el dispositivo $(q)$ y su capacidad $(Q)$; es decir, $x=q / Q$.

Se debe diferenciar el grado de saturación $(x)$ arriba definido de la denominada congestión. La segunda es consecuencia del primero y suele manifestarse en:

- Demoras adicionales a las personas, más allá de las requeridas para hacer un viaje a una velocidad segura y constante.

- Colas de vehículos o personas, que se generan en tramos de vía, cruces o terminales de transporte público o privado (paraderos, estacionamientos).

- Detenciones involuntarias de vehículos o usuarios, que ocurren en los mismos lugares anteriores, producto del avance y estancamiento de una cola (condición start-stop).

Varios estudios (Akçelik, 1981; IHT, 1997) indican que las manifestaciones de la congestión comienzan a producirse para valor es de $x$ superiores a 0,7 ó 0,8 ; o sea, cuando el flujo supera el 70 u $80 \%$ de la capacidad. Lo anterior se puede ilustrar en la Figura 2, en que se muestra la evolución de uno de los índices de congestión: la demora por usuario en un cruce.

En la figura se observa que el aumento acelerado de la demora comienza a manifestarse para $x=0,72$. A partir de ese momento un aumento del flujo, produce un aumento más que proporcional en la demora. Por ejemplo, para grados de saturación moderados, si $x$ aumenta de 0,75 a 0,80 ( $7 \%$ de variación) la demora aumenta en un $12 \%$; sin embargo, al borde de la sobresaturación, si $x$ varía de 1,00 a 1,05 (5\% de variación) la demora aumentaría en un $80 \%$. Este comportamiento se repite para los otros índices de congestión; es decir, las longitudes de cola y las detenciones. Lo mismo ocurre con los otros impactos derivados del tráfico: polución, ruido, riesgo, segregación, intimidación, intrusión visual, inaccesibilidad al transporte público, como se explica en el Capítulo 3. 
Figura 2. Variación de la demora con el grado de saturación en un cruce.

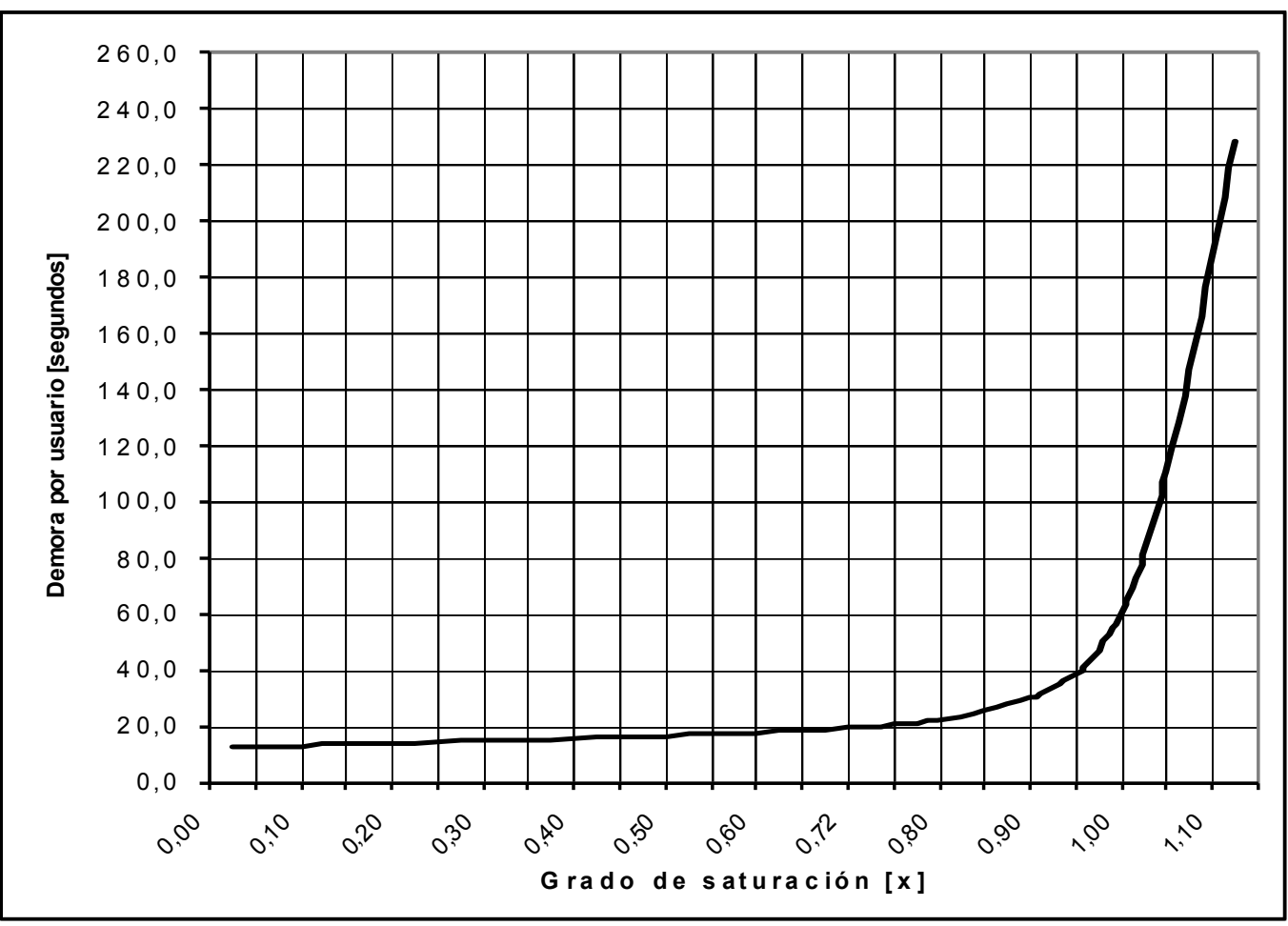

\subsection{De la teoría a la práctica}

No obstante la anterior definición de la gestión de tránsito moderna (o gestión ambiental de tránsito), es común observar -al menos en Chile- que el ámbito de acción de la ingeniería de transpor te ha derivado principalmente a atacar problemas puntuales de congestión vehicular. El énfasis se ha puesto en el rediseño de ejes viales y en la optimización de intersecciones para acomodar crecientes demandas de tráfico. O sea, la anticuada práctica de tratar de aumentar la capacidad vial y las v elocidades de desplazamiento de los autos. Los peatones no existen, tampoco los usuarios de transporte público, menos los residentes.

La actitud descrita soslaya la riqueza de herramientas de la ingeniería de transporte para abordar otros problemas urbanos y ambientales, como los accidentes de tránsito, la contaminación local, la accesibilidad al transporte público, la calidad urbana de los barrios, etc. Desconoce también la necesidad de integridad de toda gestión (al nivel de áreas, aplicando conjuntos de medidas y atacando una variedad de impactos). Además, olvida un hecho empíricamente probado: los flujos vehiculares tienen la habilidad de sobreponerse a cualquier aumento de la capacidad vial.

Se requier e por ende de una propuesta más creativa desde la ingeniería civil. No obstante, para llevar adelante esta propuesta hay algunas dificultades. Por mencionar sólo algunas:

- Despreocupación institucional por el tema. Ejemplo: ¿quién es el responsable de la única vía exclusiva para buses de Santiago?

- Falta de ar ticulación de la gestión de tránsito con los planes estratégicos de transporte y urbanismo. Ejemplo: ¿qué pasará con los flujos transferidos a calles alternativas debido al funcionamiento de una autopista urbana privada?

- Escasez de conocimiento especializado en el gobierno local -al nivel de ingeniería civil o postgrado en transporte-, además de actualizado en los últimos 5 años. Ejemplo: ¿cuántos ingenieros civiles en transporte trabajan para las municipalidades de la Re- 
gión? ¿Cuántos Alcaldes recurren a su asesoría al tomar decisiones que afectarán la circulación en su comuna?

La consecuencia es una superabundancia de proposiciones aisladas y mal concebidas porque no se ha formulado correctamente el problema que se pretenden atacar. Ejemplos van desde megalómanos proyectos de autopistas urbanas o metros elevados hasta "lomos de toro” por todas partes. Pero, ¿será fácil para el usuario de tercera edad cruzar una calle o tomar una micro?

Algunas acciones necesarias para superar estas dificultades y enfrentar el desafío de mejorar la "ingeniería de detalle en la calle" que proponemos son:

- Incluir la gestión de tránsito en la planificación urbana. Por ejemplo, partiendo por planes reguladores con una jeraquización vial basada en variables e impactos de tráfico y no sólo en el ancho de vías.

- Incorporar esquemas integrados de gestión de tránsito en los planes estratégicos de transporte. Por ejemplo, si se planifica una línea de metro, establecer cómo llegarán los usuarios a las estaciones. Si a pie, ¿son adecuadas las v eredas y cruces peatonales? Si en buses, diseñar los paraderos. Si en auto, establecer un sistema de park-and-ride (estacionamientos de larga estadía en las estaciones).

- Otorgar apoyo técnico local mediante asesoría especializada. Por ejemplo, a través de equipos técnicos itinerantes de alto nivel. Estos equipos podrían visitar varios municipios, entregar sus recomendaciones, dejar tareas y volver periódicamente a evaluar los resultados.

- Definir un organismo coordinador de la gestión de tránsito al nivel de las grandes áreas metropolitanas que fomente, haga homogénea y controle su aplicación.

En resumen, el estado de la aplicación de la gestión de tránsito en Chile es modesto y anticuado. Baste recordar que la necesidad de jerarquizar la red vial se formuló antes del término de la Segunda Guerra Mundial (Tripp, 1942) y que el concepto de capacidad ambiental de tráfico se definió hace ya 40 años (Buchanan, 1963).

\section{Los impactos y su cuantificación}

Hemos dicho que el tráfico de vehículos en las ciudades conlleva degradación de la calidad de vida urbana. Esta se ve manifestada, conforme aumenta el grado de saturación, en impactos como:

- Congestión. Aumento del tiempo de viaje, formación de colas y detenciones involuntarias.

- Riesgo. Aumento del número y gravedad de accidentes de tránsito.

- Polución. Aumento de emisiones de contaminantes atmosféricos.

- Ruido. Aumento del nivel de ruido y vibraciones en calles y edificios.

- Segregación. A umento en la distancia y tiempo de cruce de cauces vehiculares.

- Intimidación. Disminución del uso de las calles para otros fines (estar, jugar, pasear).

- Intrusión visual. Disminución del campo visual por vehículos o infraestructuras.

- Inaccesibilidad al transporte público. Dificultad para usar los servicios de transporte público.

De los impactos mencionados, la congestión ha sido tradicionalmente reconocida por todos como el principal impacto derivado del aumento del tráfico. Pero, la aprensión se reduce a considerar el efecto sobre los vehículos. La congestión peatonal o al interior de vehículos de transporte público no se vislumbra como un problema para la ingeniería ni el urbanismo. Posteriormente, frente al problema de contaminación atmosférica en las ciudades, se ha incorporado la polución del aire causada por fuentes móviles a la preocupación política. Últimamente, la alta tasa de accidentes de tránsito ha llevado a introducir también el riesgo como una de las preocupaciones de la ingeniería.

Uno de los argumentos para no considerar los restantes impactos en la gestión de tránsito y urbana ha sido la dificultad de su valoración económica y la complicación para incorporarlos en los modelos estratégicos de transporte y uso del suelo.

Para sobreponerse a las limitaciones por las cuales no se considera una mayor gama de impactos de tráfico, se presenta a continuación una forma de cuantificación física. Se ha elegido esta forma de consi- 
derar los impactos, ya que su manifestación primera sobre la urbe es física y no económica. En tal condición primordial afecta a los habitantes, siendo el resultado económico un efecto de segundo orden. De igual modo como el principal resultado de un terremoto es la destrucción y pérdida de vidas, el que luego puede valorarse en términos económicos. Por eso los terremotos se miden en magnitud Richter (1935), y no en millones de dólares, para diseñar medidas de mitigación. De este modo, se propone la siguiente cuantificación de los impactos urbanos y ambientales derivados del tráfico o la investigación necesaria para realizarla.

\subsection{Congestión}

Corresponde al aumento de tiempos de viaje a conductores y pasajeros de vehículos motorizados, así como a peatones y rodados, producto del número de usuarios que quiere simultáneamente utilizar el mismo dispositivo vial. Su medición es directa o puede ser estimada de modelos de tráfico tradicionales (ver por ejemplo, TRB, 1992; Fernández, 1999). Depende directamente del grado de saturación del dispositivo, como se mostró en la Figura 2.

\subsection{Polución}

Es el aumento de emisiones de contaminantes atmosféricos producidos por el tráfico (COV, COx, $\mathrm{NOx}$, PM10, PTS, SOx,). De estos, algunos tienen más impacto a nivel local como el monóxido de carbono (CO), el material particulado respirable (PM10) y las partículas totales en suspensión (PTS). Varios se encuentran declarados al niv el de saturación o latencia en la Región Metropolitana, como PTS, PM10, CO, O3 y NO2 (CONAMA, 1998). Existen algunos estudios que relacionan niveles de tráfico con emisiones de contaminantes (EPA, 1975; Madrid, 1998), siendo actualmente factible incorporar su cuantificación en estudios de gestión de tránsito, mediante modelos como POLAR de CUBE (Citilabs, 2003) que consideran la norma europea AUOT/OIL 1 y EPEFE (European P rgram on Emissions Fuels and Engine Technology). La emisión de contaminantes por parte de los vehículos, se explica mediante la relación siguiente (Osses et al., 2001).

$$
E_{i j k t}=q_{j k t} l_{j} F_{i k}\left(v_{j k t}\right)
$$

Donde $E_{i j k t}$ son las emisiones del contaminante $i$ en el tramo $j$ emitidas por un vehículo tipo $k$ en un perío$\operatorname{do} t(\mathrm{~g} / \mathrm{h}) ; q_{j k t}$ es el flujo por el tramo $j$ de vehículos tipo $k$ en el período $t(\mathrm{veh} / \mathrm{h}) ; l_{j}$ es la longitud del tramo $j$ $(\mathrm{km}) ; \mathrm{y} F_{i k}\left(v_{j k}\right)$ es el factor de emisión del contaminante $i$ por el vehículo tipo $k(\mathrm{~g} / \mathrm{veh}-\mathrm{km}) . F_{i k}$ es inversamente proporcional a $v_{j k r}$ que es la velocidad promedio en el tramo $j$ del vehículo tipo $k$ en el período $t(\mathrm{~km} / \mathrm{h})$. Es bien sabido que la velocidad promedio de un vehículo en un tramo disminuye con el grado de saturación (TRB, 1992). Por otra parte, si el flujo $\left(q_{j k}\right)$ de vehículos aumenta, a igual capacidad, el grado de saturación también lo hace. En consecuencia, las emisiones vehiculares aumentan al crecer $x$ por dos vías: disminución de la velocidad e incremento del flujo.

\subsection{Riesgo}

Es el aumento del número y gravedad de los accidentes de tránsito. Este impacto se puede medir a partir de las estadísticas de accidentes. Se han desarrollado modelos entre el número de accidentes en un tramo de vía $\left(A_{R}\right)$ y el flujo circulante $(q)$, mediante funciones del tipo $A_{R}=a q^{b}$. (Satterthwaite, 1981). También, como la mayoría de los accidentes de tránsito se producen en las intersecciones, Tanner (1953) propuso funciones del tipo $A_{J}=K\left(q_{1}\right)^{c}\left(q_{2}\right)^{d}$, donde $q_{1}$ y $q_{2}$ representan los flujos vehiculares de las ramas en conflicto. En todas estas expresiones, $K, a, b, c y d$ son parámetros a calibrar. Asumiendo igualdad de capacidades, las relaciones anteriores se pueden expresar como función del grado de saturación $x$.

$$
\begin{gathered}
A_{R}=a(x Q)^{b} \\
A_{J}=K\left(x_{1} Q_{1}\right)^{c}\left(x_{2} Q_{2}\right)^{d}
\end{gathered}
$$

Donde $x, x_{1}$ y $x_{2}$ son los grados de saturación de un tramo de vía y de ramas que confluyen a una intersección, respectivamente; y $Q, Q_{1}$ y $Q_{2}$ son sus respectivas capacidades.

No obstante, los resultados de estos análisis deben ser comentados. Si bien al aumentar el grado de saturación $(x)$ los accidentes pueden aumentar, como la velocidad de desplazamiento será menor, su gravedad se reduciría. Es decir, al aumentar $x$ habrá más accidentes, pero menos graves. 


\subsection{Ruido}

Es el aumento en el nivel de ruido en las calles, medido en $\mathrm{dB}(\mathrm{A})$, y vibración en edificios debido al tráfico en una vía. Existen dispositivos para medir directamente el ruido y hay modelos matemáticos que lo ligan al flujo vehicular (Department of Environment, 1978), así como estudios sobre vibraciones en edificios (Martin et al., 1978). Para la predicción del nivel de ruido en función del flujo vehicular en un tramo de vía, existen modelos del tipo $L_{10}=f+g \cdot \log (q)$. Donde $L_{10}$ es el nivel base de ruido a $10 \mathrm{~m}$ del borde de la vía, para un flujo compuesto sólo por vehículos livianos circulando a $75 \mathrm{~km} / \mathrm{h}$ sobre un pavimento en buen estado; $q$ es el volumen de tráfico en las 18 horas de un día; $f y g$ son parámetros que dependen de la velocidad, de la pendiente de la vía y de la proporción de vehículos pesados. Igual que en el caso anterior, esta expresión se puede escribir en función del grado de saturación $x$ como:

$$
L_{10}=k+g \cdot \log (x)
$$

Donde $k=(f+h)$ y $h=\log (Q)$, son constantes. $\mathrm{O}$ sea, al aumentar $x$, aumenta el nivel de ruido en las inmediaciones de la vía.

\subsection{Segregación}

Se puede estimar mediante el aumento en la distancia y tiempo de cruce de los cauces vehiculares por los peatones y rodados (bicicletas, coches para niños, carros de compras, sillas de ruedas, etc.). Si bien puede ser medida en terreno en una situación actual, se propone el siguiente método de predicción para una condición hipotética.

Supóngase una corriente ininterrumpida de flujo vehicular $q$; la segregación puede ser medida como la demora en cruzar la vía mediante brechas en el flujo. La brecha promedio (b) es el inverso del flujo vehicular; es decir, $h=1 / q$. Asumiendo la tradicional racionalidad de los usuarios de transporte, que dice que éstos utilizarán la ruta más corta para ir de un origen a un destino, los peatones tenderán a cruzar en línea recta por cualquier punto de la vía; no necesariamente en la intersección más cercana1. A medida que el flujo vehicular $q$

\footnotetext{
${ }^{1}$ Es interesante notar que, así como se acepta sin discusión este comportamiento racional en los conductores, se tiende a olvidar en el caso de los peatones, pidiéndoles que sean irracionales respecto a su elección de ruta.
}

aumenta, la disyuntiva estará entre esperar una brecha apropiada y cruzar la vía o desviarse a una ruta más larga. Esto último implica caminar hasta el cr uce regulado más cer cano, esperar por el derecho de paso (v.g., luz verde), cruzar la vía, y caminar al destino. En el primer caso, un modelo de brechas para estimar la demora de cr uce dará la medida de la segregación (ver por ejemplo TRB, 1992). En el segundo caso, la segregación puede ser medida como el tiempo de caminata en ambos lados de la vía, más la demora para cr uzar. Tanto las brechas como las demoras de cruce dependen del grado de saturación $x$, como se observa en la Figura 2 . Es decir, la segregación también será directamente proporcional al grado de saturación.

\subsection{Intimidación}

Corresponde a la disminución del uso de los espacios públicos para fines no relacionados con el transporte (estar, jugar, pasear). Existen pocos análisis cuantitativos relacionados con este tema, siendo una interesante línea de inv estigación. No obstante, una revisión realizada por Davis (1992) muestra un caso de estudio en calles de la ciudad de San Francisco, EEUU. En este estudio se analizó el nivel de relaciones sociales (amistades y conocidos) por persona en una cuadra. Se pudo determinar que si el flujo en una cuadra era de 200 vehículos por hora, el promedio de amistades era de 3,0 por persona y el de conocidos 6,3 por persona. Por el contrario, si el flujo alcanzaba los 1.900 vehículos por hora, estos números se reducían a un tercio de los anteriores ( 0,9 y 3,1 respectivamente).

\subsection{Intrusión visual}

Es la disminución del campo visual del horizonte natural por vehículos (estáticos o en movimiento) o infraestr ucturas de transporte (vías elevadas, pasos superiores, etc.). El método del ángulo sólido desarrollado por Clamp (1976) permite su cuantificación. Consiste en dos modelos, uno que permite estimar la magnitud de la intr usión pasando de una escala subjetiva a una objetiva, mediante la cuantificación de variables tales como: existencia de prados, cantidad de arbustos, existencia de bosques y casas, entre otras variables relevantes. Otro modelo permite estimar el grado de cambio antes y después de una intervención. Se puede utilizar las potencialidades de la fotografía digital e incor- 
porar dentro de una foto del paisaje el proyecto analizado.

\subsection{Inaccesibilidad al transporte público}

Es un impacto aún más ignorado. Es el aumento de la distancia, tiempo e impedancias de caminata a los puntos de acceso al sistema de transpor te público (estaciones, paraderos) producto de un aumento del tráfico en sus inmediaciones. Se están buscando medidas cuantitativas para su predicción. Algunas propuestas sugieren usar el porcentaje de usuarios que quedan impedidos de usar el transpor te público producto de barreras físicas generadas por el diseño urbano (Tyler, 1999). Por ejemplo, el cambio de ubicación de un paradero para mejorar el desplazamiento de los v ehículos particulares por una vía, puede impedir que cier tas personas puedan alcanzarlo.

Los autores han desarrollando un modelo predictivo con la finalidad de intentar cuantificar la accesibilidad del transporte público (Fernández et al., 2001). La investigación ha arrojado que dentro de las variables que contribuyen a la inaccesibilidad están: la distancia de caminata al paradero, la demora en cruzar intersecciones, la mala condición del entorno, el nivel de ruido producido por el tráfico, el mal estado e inadecuado ancho de veredas, y la percepción de inseguridad. Como se ha visto, al menos la demora de cruce, el nivel de ruido y la inseguridad son función del grado de saturación $x$. En consecuencia, este impacto también depende propor cionalmente del grado de saturación.

Tyler (1999) ha encontrado que para la tercera edad un umbral de caminata aceptable al paradero más cercano es de 2 cuadras. Aplicando este criterio a recorridos alimentadores de la Línea 5 del Metro de Santiago (EXE, 1998) se encontró que para cer ca del 30\% de la población de las comunas de San Joaquín, Macul, La Florida y La Granja, el transporte público resulta poco accesible.

\section{Aplicación}

Siempre ha existido la controversia, tanto entre ingenieros como urbanistas, respecto de la conveniencia o no de ampliar la capacidad vial para reducir "la congestión". Como se ha expuesto en este ar tículo, este es un concepto bastante nombrado, pero poco comprendido en cuanto a cuáles son sus manifestaciones físicas y cuándo se produce. Las recurrentes opiniones en pos y en contra de las autopistas urbanas son una muestra de esta polémica e incomprensión.

El siguiente ejercicio teórico intenta mostrar cómo la ingeniería de transporte, a través de una gestión de tránsito con una visión más amplia, puede complementar la toma de decisiones que afecten a la ciudad.

De la lectura del Capítulo 3 debe haber quedado claro para el lector que la mayoría de los impactos urbanos y ambientales derivados del tráfico dependen del grado de saturación $(x)$. Nos concentraremos por lo tanto en el análisis de dicha variable a escala de toda la ciudad de Santiago en el siguiente ejercicio. Para ello utilizaremos los datos de la Encuesta Origen Destino deViajes 2001 Gran Santiago -EOD 2001 (SECTRA, 2002).

Sea $P$ la suma del patrón de viajes $F$, expresada como el número total de pasajeros por hora (pax/h) que se mueve en toda la ciudad y $K$ la tasa media de ocupación de los vehículos, en pasajeros por vehículo (pax/veh). Entonces, el flujo total de vehículos se puede calcular como $q=P / K$. Por lo tanto, el grado de saturación se puede escribir como $x=P /(Q \cdot K)$. Por ende, si la disminución de $x$ trae aparejada la reducción de los impactos urbanos, ésta se puede conseguir mediante tres estrategias: (a) aumentar la capacidad de la red vial (aumentando Q); (b) aumentar la eficiencia en el uso de los vehículos (aumentando $K$ ); y (c) disminuir la demanda de viajes en el área (disminuyendo $P$ ).

Según la EOD 2001, entre las 7:30 y 8:30 horas de un día laboral se hacían 1,37 millones de viajes motorizados en Santiago en todas las direcciones. La distribución porcentual en los distintos modos o partición modal es de un 39,7\% de viajes en automóvil, $38,0 \%$ en bus, $7,0 \%$ en metro, $3,4 \%$ en taxis o colectivos y $11,9 \%$ en otros modos. Como cada viaje es realizado por una persona implican 1,37 millones de pasajeros transpor tados por hora. Dada la partición modal (39,7\% en auto y $38,0 \%$ en bus), sobre toda la red vial de superficie en el período punta de la mañana circulan $P=1,06$ millones de pasajeros por hora. De estos pasajeros, $P_{A}=540$ mil es transportado por automóviles y $P_{B}=520$ mil por buses.

Se puede asumir un valor para la tasa media de ocupación de los autos en la punta mañana de 2 pax/veh y 
que toda la flota de buses ( 8 mil vehículos) es utilizada en ese período. Por lo tanto, la tasa media de ocupación de los buses sería $65 \mathrm{pax} / \mathrm{veh}$. Con estos valores los flujos en cada modo, expresados en miles de vehículos equivalentes por hora (veq/h), son $q_{A}=270$ y $q_{B}=16$. $\mathrm{O}$ sea, el flujo total en la red es $q=286 \mathrm{mil} \mathrm{veq} / \mathrm{h}$. El vehículo equivalente es una abstracción para transformar el flujo de distintos tipos de vehículos en flujo de vehículos livianos homogéneos; así, 1 auto $=1$ veq y 1 bus o camión $=2$ veq.

Por lo tanto, si la capacidad de la red, expresada en miles de vehículos por hora, es $Q$, su grado de saturación será $x=286 / Q$. Este será nuestro grado de saturación de referencia. Probemos ahora las estrategias enunciadas más arriba para disminuir $x$.

\subsection{Aumento de la capacidad vial}

Según la EOD 2001, entre 1991 y 2001 el patrón de viajes creció a una tasa de un $4 \%$ al año, pero se produjo un mayor aumento de los viajes en auto que en bus, lo que modificó la partición modal a las cifras actuales. Con estos antecedentes, se puede estimar una tasa promedio anual de crecimiento de los viajes en auto del 9,4\%. En tanto, los viajes en bus no aumentaron; incluso, disminuyeron levemente. De mantenerse la misma tendencia de crecimiento difer enciado de la demanda por auto y por buses, la proyección del flujo total sobre la red en el tiempo podría expresarse a partir de los valores del 2001 como $q(t)=270(1,094)^{t}+16$, donde $t$ es tiempo expresado en años.

Con esta proyección de $q$ en el tiempo, para mantener el grado de saturación $x$ en los niveles actuales y, por ende, no empeorar los impactos urbanos más de lo que están ahora, la capacidad $Q$ de la red debía crecer en la misma proporción. En particular, cuando el flujo sea igual al doble del actual $(2 q)$, la capacidad de la red vial debería duplicarse. De la expresión de $q(t)$ se puede calcular que esto debería ocurrir cada $t=8$ años. Lo anterior significa, por ejemplo, ensanchar al doble el número actual de pistas de las principales arterias de la ciudad (Alameda, Providencia, Costanera, Irarrázaval, Vicuña Mackenna, Santa Rosa, Recoleta, Independencia, etc.) y desnivelar todos sus principales cruces dentro de los 8 años siguientes al 2001. O sea, antes del Bicentenario de la República. Y lo mismo repetirlo antes de los 8 años subsiguientes (2017), y así sucesivamente. Parece una estrategia no sostenible en el largo plazo, por sus costos económicos, urbanísticos y am- bientales, tener, por ejemplo, que construir una vía oriente-poniente de las características del eje Alameda-Providencia-Apoquindo ( 3 a 5 pistas por sentido) cada 8 años. Por eso, se deben explorar estrategias de gestión de tráfico (aumentar $K$ o disminuir $P$ ).

\subsection{Aumento de la eficiencia de uso de los vehículos}

Esta estrategia puede tener dos componentes: (a) traspaso de usuarios de autos al transporte público; y (b) aumento de la tasa de ocupación de los autos.

Para simplificar el análisis, el efecto de estas alternativ as se estudiaráceteris paribus para un corte temporal al año de la EOD 2001.

\subsubsection{Traspaso de usuarios al transporte público}

Supóngase que por alguna medida de mejoramiento del transporte público (por ejemplo, Transantiago), el $25 \%$ de los actuales usuarios de autos se traspasan a los buses. Esto implica que la demanda horaria total se mantiene en $P=1,06$ millones pax $/ \mathrm{h}$, pero se modifica su distribución modal a $P_{A}=410 \mathrm{mil} \mathrm{pax} / \mathrm{h}$ en auto y $P_{B}=$ $650 \mathrm{mil}$ pax/h en bus. Esto significa un cambio en la partición modal a un $61 \%$ en buses y $39 \%$ en auto. Es decir, parecida a la que había 10 años atrás. Si se mantienen las tasas medias de ocupación de los vehículos, los nuevos flujos horarios resultan $q_{A}=205 \mathrm{mil} \mathrm{veq} / \mathrm{h}$ y $q_{B}=$ $20 \mathrm{mil} \mathrm{veq} / \mathrm{h}$. Por lo tanto, el flujo total sobre la red disminuye a $q=225 \mathrm{mil} \mathrm{veq} / \mathrm{h}$.

En consecuencia, el nuevo grado de saturación será $x^{\prime}=225 / Q$. Comparado con la situación base $(x=286 /$ $Q$ ), significa una reducción del $21 \%$ en el grado de saturación y, por ende, en los impactos derivados del tráfico, sin necesidad de expandir la capacidad vial.

\subsubsection{Aumento de la tasa de ocupación de los autos}

Supóngase ahora que, debido a la implantación de la tarificación vial en Santiago, se hace un mejor aprovechamiento de los autos y su tasa de ocupación aumenta en un $25 \%$, alcanzando los 2,5 pax/veh. La demanda horaria total $(P)$ y las de cada uno de los modos $\left(P_{A}\right.$ y $\left.P_{B}\right)$ permanecen idénticas, es decir, la partición 
modal actual no varía. En este escenario el flujo de automóviles disminuye a $q_{A}=216 \mathrm{mil} \mathrm{veq} / \mathrm{h}$ y el flujo de buses se mantiene en $q_{B}=16 \mathrm{mil} \mathrm{veq} / \mathrm{h}$. En tales condiciones, el flujo total sobre la red es $q=232 \mathrm{mil} \mathrm{veq} / \mathrm{h}$.

Como resultado, el nuevo grado de saturación es $x$ " $=232 / Q$. Con relación a la situación base, implica una disminución del grado de saturación y efectos asociados de un $19 \%$, sin obligación de ampliar la capacidad vial.

\subsection{Disminución de la demanda de viajes}

La EOD 2001 indica que un 36\% de los viajes en el período punta de la mañana son por motivo de estudio. La razón es que la generalidad de los colegios se encuentra en la zona centro-oriente de la ciudad, en tanto que las may ores densidades residenciales se sitúan en la zona sur-poniente. Además, no hay homogeneidad en la calidad educacional entre los distintos barrios, lo que induce viajes de escolares más allá de su entorno local.

Supóngase que por una homogeneización en la localización y calidad de los centros educacionales, se disminuyera en un $50 \%$ la demanda de viajes por estudio. Esto implicaría un $18 \%$ de disminución del total de viajes en hora punta a 1,16 millones de pax/h. A igualdad de todas las otras variables, la demanda de viajes en la red vial superficial sería $P=901 \mathrm{mil} \mathrm{pax} / \mathrm{h}$. Las respectivas demandas y flujos en autos y buses en las misma unidades usadas más arriba son: $P_{A}=461, \mathrm{q}_{\mathrm{A}}=231$, $P_{B}=440, q_{B}=14$. Por consiguiente, el flujo total en la red se reduciría a $q=245 \mathrm{mil}$ veq $/ \mathrm{h}$.

Por tanto, el grado de saturación de la red sería $x^{\prime \prime}=$ $245 / Q$. Es decir, un $14 \%$ de disminución con respecto a la situación actual, para igual capacidad vial.

\subsection{Comentarios}

En cada una de las alternativas anteriores se observan disminuciones no despreciables del grado de saturación de la red sin tener que aumentar su capacidad, sino que con un mejor aprovechamiento del sistema de transporte $(T)$ actual o una modificación del sistema de actividades $(A)$. Hay que destacar que reducciones del
14 al $21 \%$ de los flujos vehiculares en hora punta son más de lo que se ha logrado nunca con la restricción vehicular en Santiago.

Esta aplicación muestra que, si se aplicaran políticas que incentiven este tipo de respuestas (mejoramiento del transporte público, tarificación vial, creación de buenos colegios públicos), la actual presión por grandes expansiones en la capacidad de la red vial en el mediano plazo se aliviaría. En el corto plazo, el conjunto de los impactos urbanos y ambientales derivados del tráfico se podría reducir en proporción a la disminución del grado de saturación $(x)$, como lo hemos demostrado mediante las ecuaciones (1) a (4). Por ejemplo, podrían esperarse reducciones en tiempos de viaje, contaminación atmosférica, accidentes, ruido, etc. No es por casualidad que las actuales políticas de transporte en el mundo desarrollado se orienten a este tipo de iniciativas, en lugar de a la expansión de la capacidad de la red vial (ver por ejemplo DETR, 1998).

\section{Conclusiones}

La incorporación de una perspectiva más amplia de la ingeniería de transporte puede ayudar a una mejor gestión urbana y ambiental. Esta perspectiva es hoy día factible, existiendo diversos enfoques para evaluar otros impactos urbanos derivados del tráfico, aparte de la sola congestión vehicular. Se puede especular, entonces, que la ausencia de tal perspectiva nace de un desconocimiento técnico y político de las potencialidades que tiene la gestión de tránsito en la solución de los problemas urbanos. Por lo tanto, la intención de este artículo es contribuir a superar tal desconocimiento y enriquecer la discusión en este campo. Debe entenderse como introductorio al tema. En ningún caso ha agotado el análisis.

La aplicación de una gestión ambiental de tránsito como la expuesta debería conducir a un cambio en las políticas urbanas y de transporte. Desde ver el problema como "la congestión", para el cual la solución es el aumento de la capacidad vial, a considerar que el problema es la calidad urbana, para la cual las soluciones pasan por considerar la capacidad ambiental (Buchanan, 1963) y la capacidad de transporte de personas (Fernández \& Planzer, 2002). 


\section{Referencias bibliográficas}

Akçelik, R. (1981). Traffic signals: capacity and timing analysis. Research Report ARR 123, Australian Road Research Board.

Buchanan, C. (1963). Traffic in towns: a study of the long term problems in urban areas. London: HMSO.

Citilabs (2003). CUBE. www.citilabs.com

CONAMA (1998). Plan de prevención y descontaminación atmosférica de la Región Metropolitana. Santiago: Comisión Nacional del Medio Ambiente.

Clamp, P. E. (1976) Evaluation of the impact of roads on the visual amenity of rural areas. Department of Environment. Research Report 7. London: HMSO.

Davis, A. (1992). "Livable streets and perceived accident risk: quality-of-life issues for residents and vulnerable road-users". Traffic Engineering and Control, 33, 6: 374-387.

Department of Environment. (1978). Calculation of road traffic noise. London: HMSO.

DETR (1998). A new deal for transport. Better for everyone. A summary of the Government's White Paper. London: UK Department of Environment, Transport and the Regions.

EPA (1975). Compilation of air pollutant emission factors. Washington: US Environment Protection Agency.

EXE Ingeniería \& Software Ltda. (1998). Estudio de servicios de transporte combinado-Linea 5. Santiago: Gerencia de Ventas y Servicios al Cliente, Departamento de Transporte Combinado, METRO S.A.

Fernández, R. (1999). Gestión de tránsito urbano. Publicación DIT-DOC/01/99, Departamento de Ingeniería Civil, Universidad de Chile, Santiago.

Fernández, R., R. Planzer y C. Palma (2001). "Modelos para estudiar la accesibilidad y acceso al sistema de transporte público. Actas del X Congreso Chileno de Ingeniería de Transporte. Concepción.

Fernández, R. \& R. Planzer (2002). "On the capacity of bus transit systems". Transport Reviews, 22, 3: 267-293.
IHT (1997). Transport in the urban environment. London: The Institution of Highway and Transpor tation.

Madrid, L. (1998). Estimación de emisiones de contaminantes atmosféricos en intersecciones semaforizadas. Memoria para optar al Título de Ingeniero Civil, Universidad de Chile.

Manheim, M. L. (1984). Fundamental of transportation systems analysis. Volume 1: basic concepts. Cambridge, MA: The MIT Press.

Martin, D. J., P.M. Nelson y R. C. Hill (1978). Measurement and analysis of traffic-induced vibration in buildings. Report SR 402. Transport and Road Research Laboratory, Crowthorne, Berkshire.

Osses, M., C. Gherardelli y F. Fresard (2001). “A model to determine primary air pollutant emissions from urban buses in Santiago de Chile". Proceedings of the 7th International Conference on Urban Transport and the Environment, Lemnos Island, Greece.

Richter, E. F. (1935). "An instrumental earthquake magnitude scale". Bulletin of Seismological Society of America, 25: 1-32.

Satterthwaite, S. P. (1981). A survey of research into relationships between traffic accidents and traffic volumes. Report SR 692. Transport and Road Research Laboratory, Crowthorne, Berkshire.

SECTRA (2002). Encuesta Origen Destino de Viajes 2001 Gran Santiago. Comisión de Planificación de Inversiones en Infraestructura de Transporte, Secretaría Ejecutiva, Santiago (http://www.sectra.cl/ transporte/transporte_urbano_eod_frm.html).

Tanner, J. C. (1953). "Accidents at rural three-way junction". Journal of the Institution of Highway Engineers, 2, 56-67.

TRB (1992). Traffic flow theory-a state-of-the-art report. Washington, DC: Transportation Research Board, Federal Highway Administration.

Tripp, A. (1942). Town planning and road traffic. London: Arnold.

Tyler, N. A. (1999). "The APEX measure: concepts”. University of London Centre for Transport Studies Working Paper. 